\title{
Signs of RV overload on the athlete's ECG
}

\author{
Guido Claessen, MD, ${ }^{\mathrm{a}, *}$ Maria Brosnan, MD, ${ }^{\mathrm{b}}$ \\ Andre La Gerche, MD, PhD, ${ }^{\mathrm{a}, \mathrm{c}}$ Hein Heidbuchel, MD, PhD ${ }^{\mathrm{d}}$ \\ ${ }^{\text {a }}$ Department of Cardiovascular Medicine, University Hospitals Leuven, Leuven, Belgium \\ ${ }^{\mathrm{b}}$ St Vincent's Hospital Department of Medicine, University of Melbourne, Fitzroy, Australia \\ ${ }^{\mathrm{c}}$ Baker IDI Heart and Diabetes Institute, Melbourne, Australia \\ ${ }^{\mathrm{d}}$ Hasselt University and Heart Center, Jessa Hospital, Hasselt, Belgium
}

\begin{abstract}
There is increasing evidence that regular intense endurance exercise can promote structural and electrical remodeling of the right ventricle (RV). These physiological changes can be profound and are frequently accompanied by ECG changes in the right precordial leads, thereby mimicking features observed in arrhythmogenic right ventricular cardiomyopathy (ARVC). Because the 12-lead ECG is used as both a screening and diagnostic tool for the detection of conditions associated with sudden death in athletes, it is of fundamental importance to have a good understanding of the ECG features that distinguish physiological adaptations to endurance exercise from those related to RV pathology as well as their potential overlap. This article describes ECG findings observed in healthy endurance athletes versus athletes with underlying RV pathology and illustrates their differentiation using 4 case presentations. (C) 2015 Elsevier Inc. All rights reserved.

Keywords: Right ventricle; Electrocardiographic; Cardiomyopathy; T-wave inversion
\end{abstract}

\section{Introduction}

It has long been known that regular, intensive endurance training induces a combination of electrical, structural and functional cardiac alterations, commonly referred to as athlete's heart [1]. As a result, the vast majority of highly trained endurance athletes demonstrate training-related changes on the resting 12-lead electrocardiogram (ECG) [2]. Although these adaptations are generally considered the expression of adaptive physiology in athletes, it is often challenging to reliably distinguish these from abnormal ECG findings related to potentially lethal cardiovascular conditions due to their phenotypic overlap. For this purpose, ECG interpretation guidelines classify ECG changes as either those which are common and training related ("group 1") or those thought to be uncommon, training unrelated, and which warrant further investigation to exclude cardiac pathology ("group 2") $[3,4]$. Nevertheless, recent data have shown that even group 2 ECG findings are not so uncommon in highly-trained endurance athletes, particularly those ECG features suggestive of right ventricular (RV) pathology such as deep T-wave inversion (TWI) in the right precordial leads and voltage criteria for RV hypertrophy [2]. This can be

\footnotetext{
* Corresponding author at: Department of Cardiovascular Medicine, University Hospitals Leuven, Herestraat 49, B-3000 Leuven, Belgium Europe.

E-mail address: guido.claessen@uzleuven.be
}

explained by more profound structural and/or electrical right ventricular remodeling in endurance athletes. Indeed, it has been shown that they develop greater RV than left ventricular (LV) remodeling which has been linked to the greater hemodynamic load that is put on the RV during intense exercise [5]. Therefore, particularly in endurance athletes, it can be challenging to differentiate benign physiological ECG changes from those observed in RV pathology such as arrhythmogenic right ventricular cardiomyopathy (ARVC).

The aim of this paper is to provide an overview of ECG features expressing RV physiological adaptation to regular endurance training and how to differentiate these from ECG findings related to underlying RV pathology. We will illustrate this clinical conundrum using 4 case examples. These cases were selected from an existing cohort at our institution to highlight different aspects of the evaluation and management of athletes with (potential) RV pathology.

\section{Overview of ECG abnormalities in athletes with RV pathology}

ARVC is an inherited heart muscle disease characterized by progressive dilation/dysfunction of predominantly the RV (and the LV in late-state disease). When evaluating the athlete's ECG it is very important to recognize abnormalities 
that point towards a diagnosis of ARVC as endurance sports continuation in a subject with ARVC may trigger life threatening arrhythmias and cause progression of the arrhythmogenic substrate [6,7]. Mutations in the desmosomal genes account for approximately $50 \%$ of ARVC cases [8]. However, there is emerging evidence that intense endurance sports may lead to a similar phenotype (with similar prognosis) even in the absence of desmosomal mutations [7,9]. This syndrome, so-called "exercise-induced ARVC" or "gene-elusive ARVC" may be the result of increased RV wall stress during exercise.

More than $80 \%$ of ARVC patients will have abnormalities on the ECG $[10,11]$. Repolarization abnormalities, such as TWI in the anterior precordial leads and delayed $\mathrm{S}$ wave upstroke, are early and sensitive markers of disease expression in patients with ARVC. T-wave inversion in $\mathrm{V}_{1}, \mathrm{~V}_{2}$, and $\mathrm{V}_{3}$ and beyond (in the absence of complete right bundle branch block, RBBB) is a major diagnostic criterion for ARVC, while TWI confined to just leads V1 and V2 represents a minor diagnostic criterion [12,13]. However, TWI in the right precordial leads, which is observed in only $4 \%$ of healthy women and $1 \%$ of men $>14$ years of age [12], is present in around $14 \%$ of highly trained endurance athletes [2]. Therefore, although right precordial repolarization anomalies are probably normal manifestations of intensive endurance training, related to the right ventricular loading conditions during sports, these create difficulties in the differentiation from early stage ARVC. We will illustrate this relatively common clinical scenario by presenting 2 endurance athletes (case 1 and 2) with a similar degree of right precordial TWI on the resting ECG and in whom further evaluation and work-up were required to differentiate physiological RV remodeling from ARVC. We will discuss electrical changes on the ECG, signal-averaged ECG and Holter. Later, we will also provide an example of an endurance athlete with more advanced disease (case 3 ) in whom both electrical and structural abnormalities are more profound.

Apart from ARVC, ECG abnormalities in the right precordial leads may also result from other conditions causing RV overload. Pulmonary hypertension is a hemodynamic state defined by a mean pulmonary artery pressure greater than or equal to $25 \mathrm{mmHg}$ and can be caused by a variety of etiologies [14]. ECG abnormalities in PH are related to physiological and anatomic adaptions of the right heart in response to increased afterload. These findings include right ventricular hypertrophy (RVH) according to the Sokolow-Lyon voltage criterion $(\mathrm{R}-\mathrm{V} 1+\mathrm{S}-$ V5 $>10.5 \mathrm{~mm}$ ), right axis deviation, TWI, ST-segment depression and right atrial enlargement $[13,15]$. In adults with idiopathic pulmonary arterial hypertension, $87 \%$ demonstrated $\mathrm{RVH}$ and $79 \%$ demonstrated right axis deviation [15]. Current European Society of Cardiology guidelines recommend that Sokolow-Lyon RVH should prompt further assessment for 'pathological RV dilatation/ hypertrophy' in young athletes [3]. Nevertheless, a recent study in asymptomatic young athletes demonstrated that Sokolow-Lyon RVH was present in around one in eight athletes, indicating that such a strategy would be associated with an unacceptably high rate of false positive screening outcomes [16]. In an attempt to reduce the number of false positives, the more recently proposed Seattle criteria for athlete ECG interpretation suggest that Sokolow-Lyon RVH should be accompanied by right-axis deviation $>120^{\circ}$ before further investigation is initiated [13]. In the fourth case of this paper, we will discuss the evaluation and management of an endurance athlete presenting with ECG features of RV hypertrophy.

\section{Case 1}

An asymptomatic 21 year old female elite rower of Caucasian origin underwent ECG pre-participation screening. She performed regular intense endurance exercise training for 7 years. The resting ECG revealed deep TWI in V1-V2 and biphasic TWI in V3, and a rightward QRS axis (Fig. 1A). Another potentially important feature on the 12-lead ECG was the notion of a slight deflection in V1, perhaps raising the query of an epsilon wave, a major ECG criterion for ARVC. However, epsilon waves are defined as reproducible waves of small amplitude that occupy the ST segment in the right precordial leads $(\mathrm{V} 1-\mathrm{V} 3)$ and are distinct from the QRS complex [17]. Accordingly, the discrete notch in the upstroke of the S-wave, i.e. part of the QRS complex, isolated to lead V1 was not considered to be an epsilon wave. Furthermore there were no other depolarization/conduction abnormalities suggestive of ARVC such as QRS prolongation in the right precordial leads $>110 \mathrm{~ms}$ or prolonged terminal activation duration of QRS $\geq 55 \mathrm{~ms}$ (measured from the nadir of the $\mathrm{S}$ wave to the end of the QRS).

Further investigations were performed to exclude underlying ARVC. Echocardiography showed a very heavily trabeculated RV with apparent hypokinesia of the RV apex (Fig. 1B). CMR confirmed a dilated, heavily trabeculated RV, but showed normal systolic function and no regional abnormalities. A signal averaged ECG (SAECG) could not identify any late potentials. Therefore, after thorough investigation, the athlete did not meet Task Force Criteria for ARVC and the diagnosis was felt to be very unlikely [12]. Furthermore, she was entirely asymptomatic, with no family history of sudden death or ARVC, and as such was not restricted from sports participation. However, given the extent of the ECG changes and RV remodeling she will be followed up annually with history-taking, physical examination and imaging.

This case illustrates a relatively common clinical scenario for physicians responsible for the cardiovascular care of athletes. Around 1 in 7 highly trained endurance athletes have been observed to demonstrate inverted $T$ waves in right precordial leads, with 1 in 25 demonstrating biphasic TWI extending into lead $\mathrm{V} 3$, thereby constituting a major criterion for the diagnosis of ARVC [2,12]. This suggests that these changes are not exclusively 'training unrelated,' but in fact reflect increased structural and electrical RV remodeling in these athletes. Nevertheless, in accordance with the 2010 Task Force Criteria and Seattle criteria, TWI involving at least two consecutive leads, excluding V1, should prompt comprehensive evaluation including echocardiography, Holter monitoring, SAECG, maximal exercise-ECG and 
A

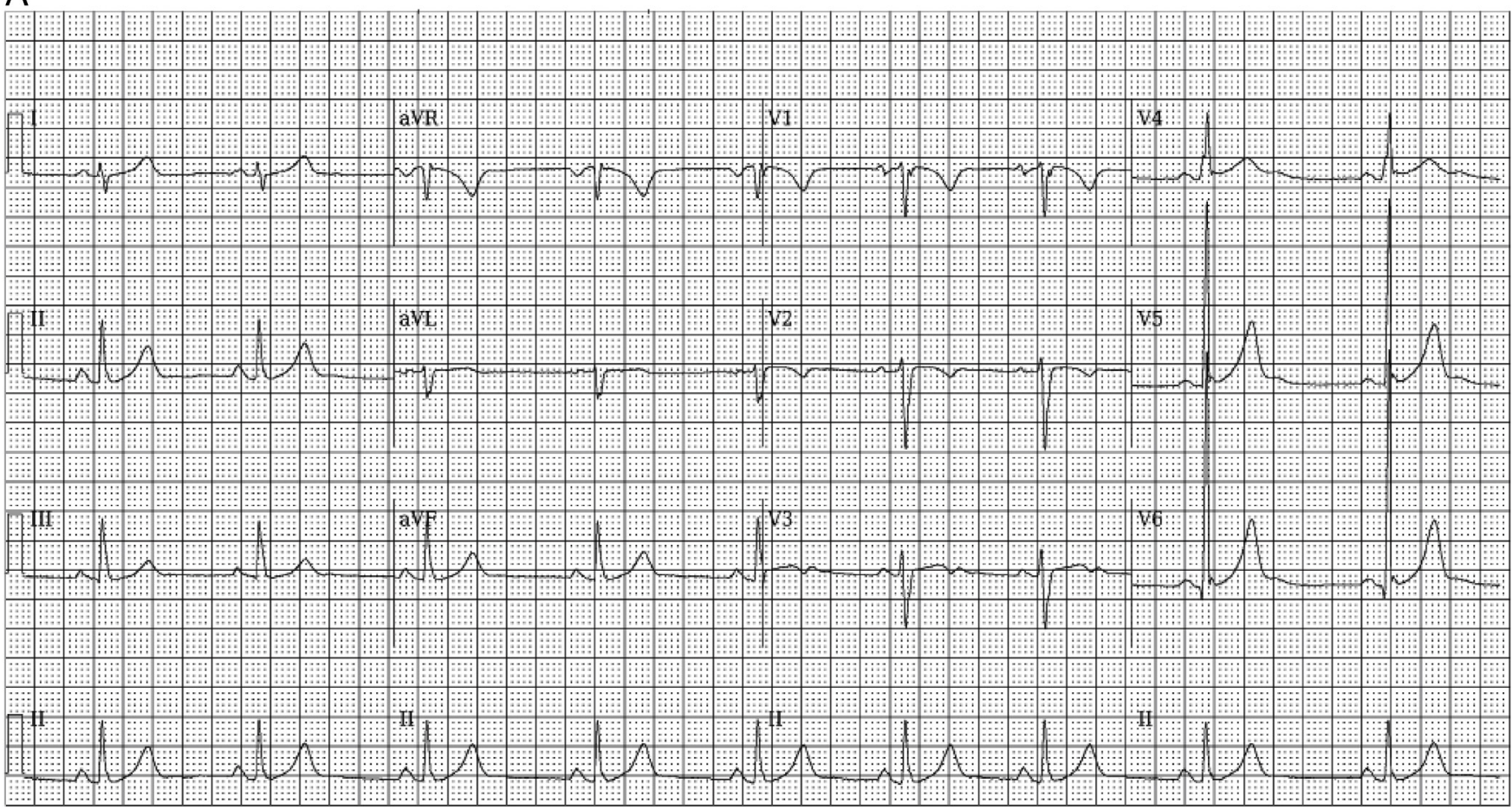

B
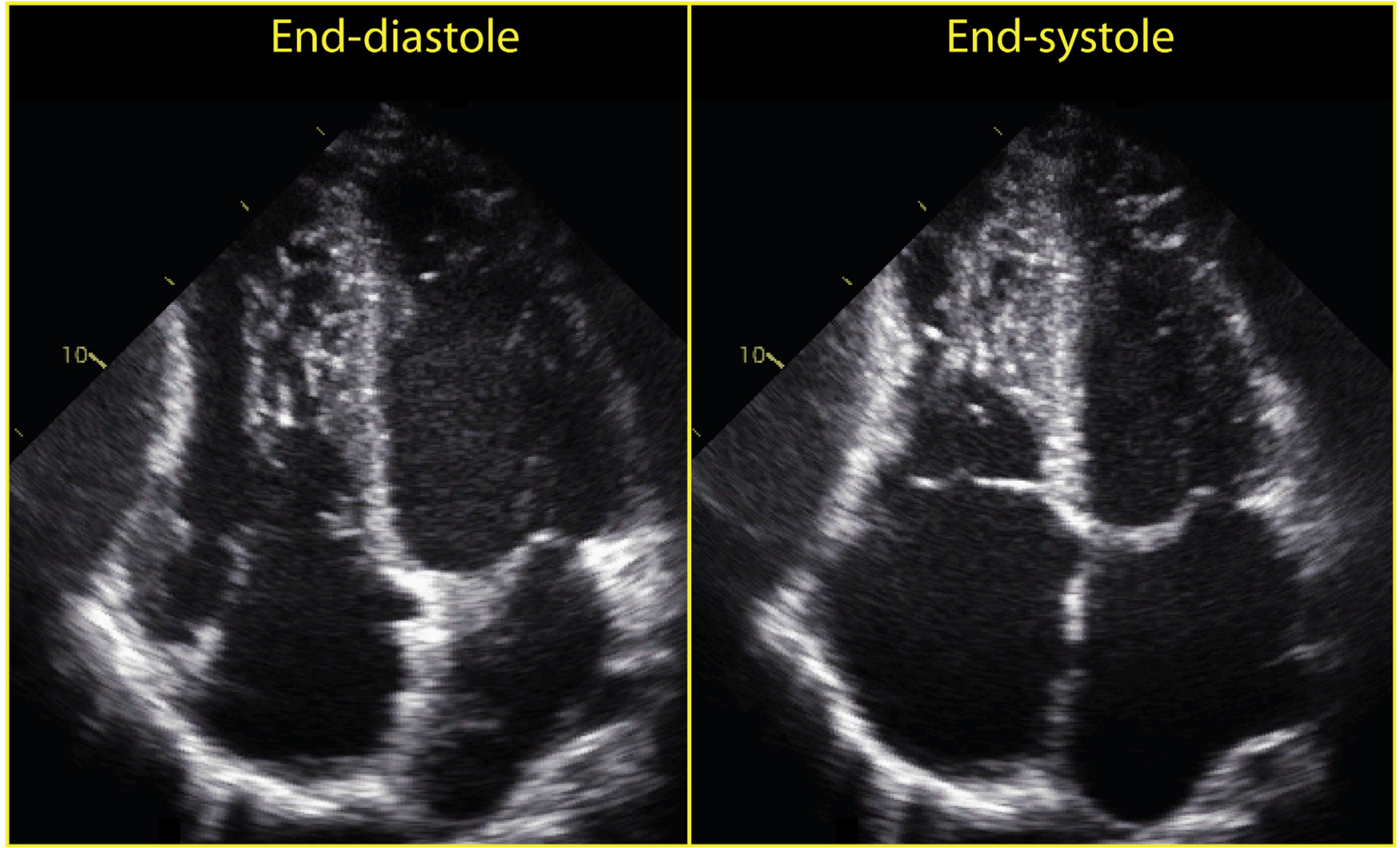

Fig. 1. Resting ECG and echocardiography in athlete 1. (A) ECG in case 1 showing deep T-wave inversion (TWI) in V1-V2 and biphasic TWI in V3. Also a deflection was noted in V1 which would raise the query of an epsilon wave. (B) Echocardiography showed a very heavily trabeculated RV with apparent hypokinesia of the RV apex.

possibly a cardiac MRI to exclude ARVC $[12,13]$. Furthermore, it is also important to consider ethnicity when interpreting TWI in the right precordial leads. Convex ST-segment elevation with biphasic TWI in leads V1-V4 is present in 1 in 7 black athletes and appears to be a benign finding in the absence of other relevant clinical indicators [18]. Conversely, both in black athletes and Caucasian athletes, the presence of deep, symmetrical TWI in all 3 leads 
from V1 to V3, preceded by isoelectric or downsloping ST segments, is rare and should prompt further investigation to exclude ARVC [2,18].

\section{Case 2}

A 22 year old professional cyclist of Caucasian origin presented with palpitations without any other accompanying symptoms such as syncope or dyspnea. The resting ECG was relatively unremarkable for a well-trained endurance athlete with TWI in V1 and biphasic TWI in V2 in the absence of any other abnormalities (Fig. 2A). In fact, when comparing the resting ECG in the first 2 cases, the extent of TWI in the right precordial leads was less remarkable in the latter and would have required limited further investigation [13], had it not been for his presenting symptoms. Holter monitoring revealed multiple runs of non-sustained VT (up to 8 beats at $220 \mathrm{bpm}$ ) during exercise. Echocardiography showed a mildly dilated RV with mildly reduced global systolic function. An electrophysiological study was performed and revealed non-sustained polymorphic VT with stimulation from the RV apex, which was considered a nonspecific finding. Since VT was only documented during exercise, treatment was initiated with a beta blocker and avoidance of sports.
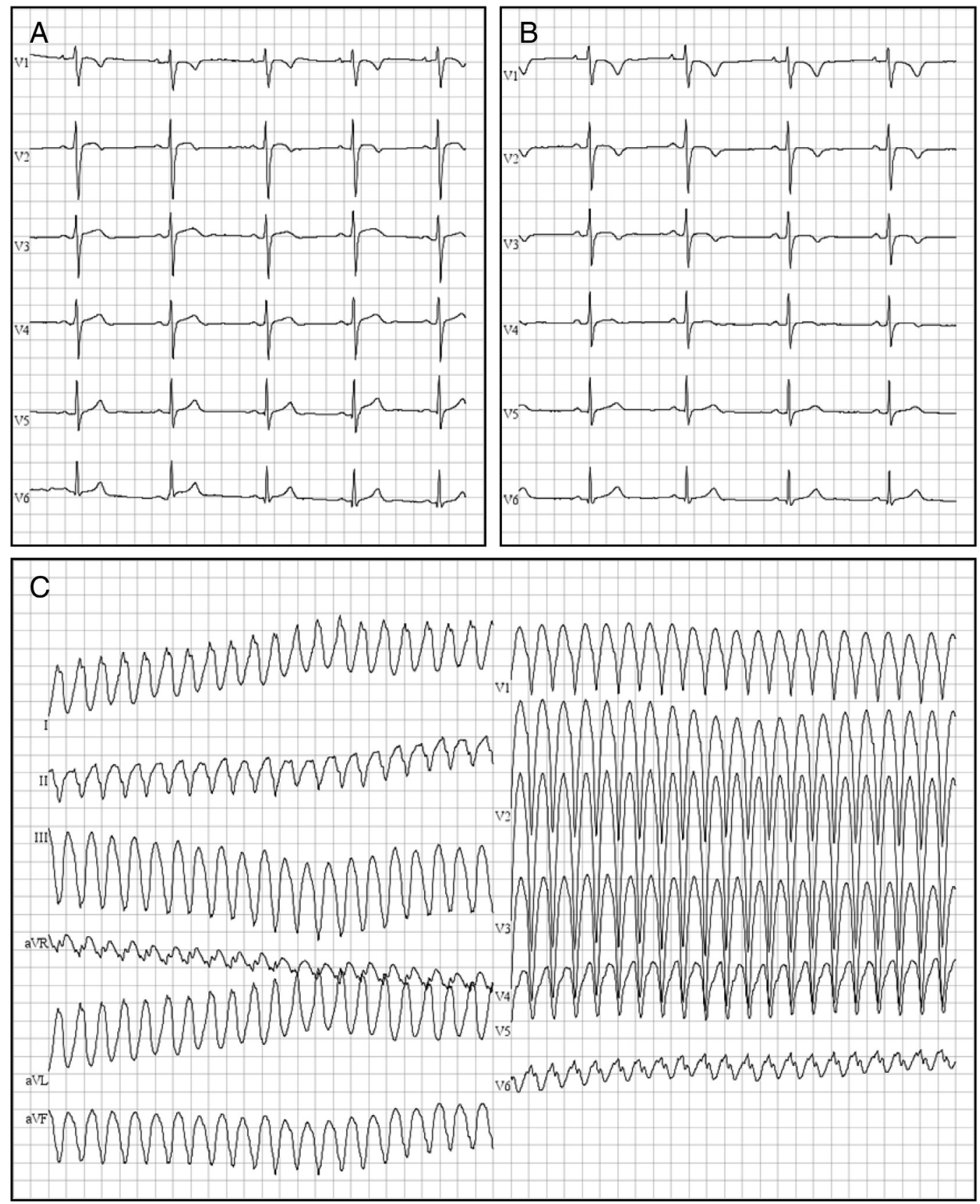

Fig. 2. ECG evaluation at initial presentation and during follow-up in athlete 2. The resting ECG at initial presentation depicts T-wave inversion (TWI) in V1 and a biphasic T-wave in V2 (A). Re-evaluation during follow-up shows development of TWI in V1-V3 (B) and episode of sustained monomorphic VT (250 bpm) with left bundle branch block morphology and right superior axis (C). 
During follow-up, there was progression of the ECG abnormalities with development of TWI in V1-V3 (Fig. 2B). Several years later, he presented to the emergency department with sustained monomorphic VT $(250 \mathrm{bpm})$ with LBBB morphology and right superior axis (Fig. 2C), requiring electrical cardioversion because of hemodynamic compromise. At this point, re-evaluation was performed. SAECG was positive for late potentials (3/3 time domain criteria; Fig. 3A). Traditionally, it has been common practice to state that the SAECG is positive if 2 of the 3 variables are abnormal (filtered QRS duration $\geq 114 \mathrm{~ms}$, duration of terminal QRS $<40 \mu \mathrm{V}$ $\geq 38 \mathrm{~ms}$ and root-mean-square voltage of terminal $40 \mathrm{~ms}$ $\leq 20 \mu \mathrm{V}$ ) [12]. However, the sensitivity and specificity of any one of the time domain criteria are similar to that of any 2 or 3 of these criteria and, therefore, any one of the criteria is considered a minor criterion for ARVC [19].

CMR imaging revealed severe dilatation of RV with reduced RV systolic function (RV EF 34\%) and regional dysfunction of the RVOT and the inferolaterobasal RV wall, whilst LV function was normal. An EP study was repeated with induction of sustained VT at $225 \mathrm{bpm}$, for which radiofrequency catheter ablation was performed and an ICD was implanted. The athlete had a negative family history of ARVC and genetic analysis for any of the five desmosomal genes was negative, both for manifest mutations and for uncommon variants of uncertain significance.
It is possible that participation in strenuous endurance exercise may have promoted progression of the arrhythmogenic substrate in this athlete. Hence, we have previously proposed the term "exercise-induced RV cardiomyopathy" to reflect the fact that endurance athletes have a propensity to develop RV remodeling consisting of structural, functional and electrophysiological changes which predispose to RV arrhythmias even in the absence of familial disease $[9,20]$. However, the discussion as to whether or not this syndrome represents a part of the spectrum of ARVC is beyond the scope of this article and the implications for sports participation are similar for the patient. Indeed, also in subjects with a genetic predisposition for ARVC, frequent endurance exercise has been shown to increase the likelihood of diagnosis, ventricular arrhythmias and development of heart failure [21]. Thus, the distinction between ECG changes as a sign of physiological $\mathrm{RV}$ remodeling versus an early sign of ARVC is of crucial importance for the management of these individuals as endurance sports continuation by itself may promote further progression of the disease.

\section{Case 3}

A 21 year old Caucasian male athlete engaged in competitive, non-professional cycling presented with pre-
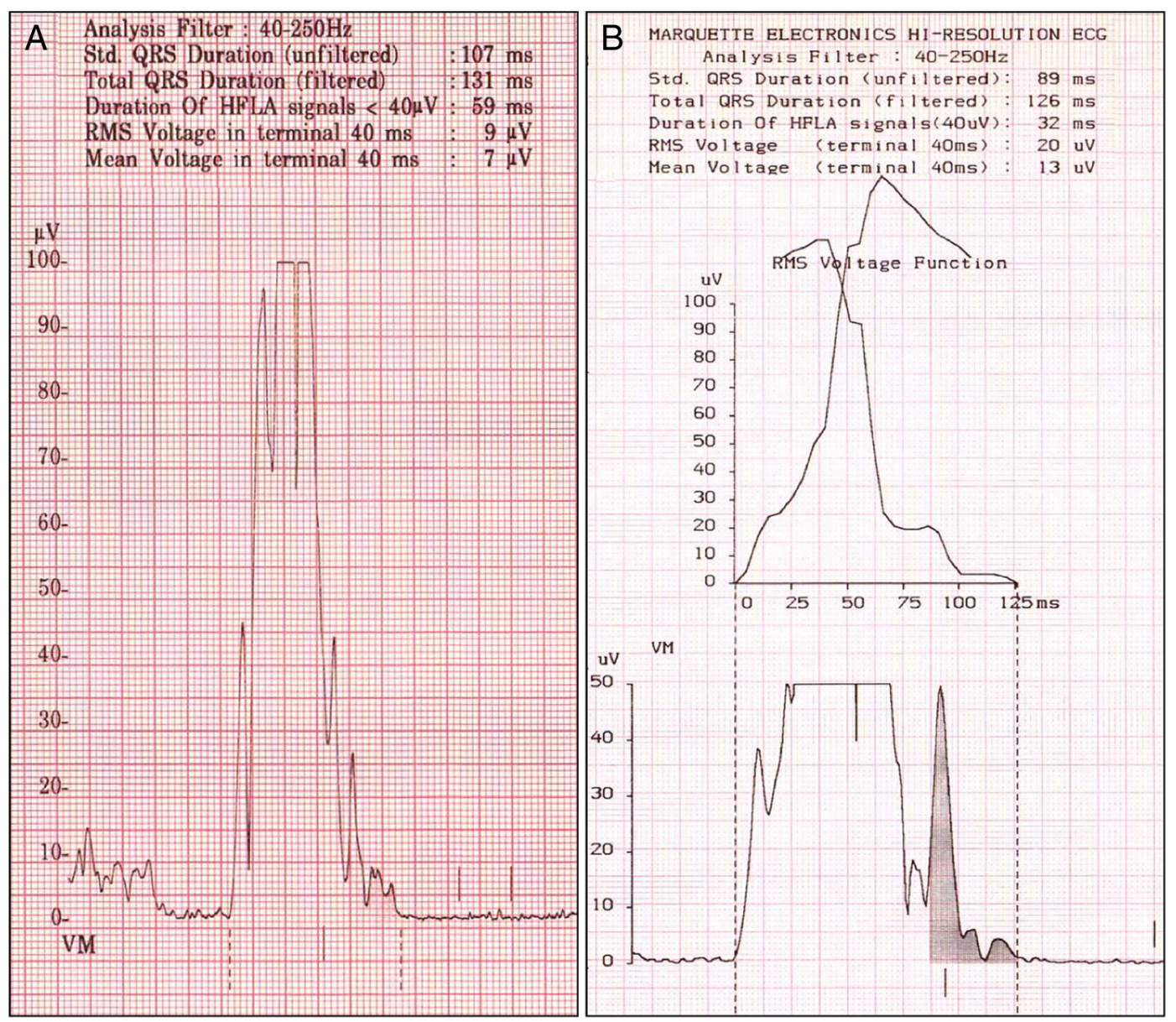

Fig. 3. Signal averaged ECG in athletes 2 and 3. Late potentials are present in athlete 2 (A) and 3 (B) on the basis of $3 / 3$ and $2 / 3$ positive criteria, respectively. 
syncope during exercise. He reported a discrete reduction of his exercise capacity over the last year. The resting ECG showed deep TWI in V1-V5, prolongation of the terminal activation duration $\geq 55 \mathrm{~ms}$ measured from the nadir of the $\mathrm{S}$ wave to the end of the QRS complex in V1 and the presence of 2 ventricular ectopic beats with LBBB morphology and superior axis (Fig. 4). SAECG demonstrated positive late potentials (Fig. 3B). During exercise ECG testing, 2 runs of non-sustained VT with LBBB morphology were observed. Sustained VT with similar morphology (LBBB and right axis) was easily induced during a subsequent EP study. CMR imaging showed mild to moderate dilation of the RV with hypokinesia of the RVOT, but no bulging or dyskinesia. An ICD was implanted for the diagnosis of ARVC. Genetic analysis was performed and revealed a conservative mutation, poorly conserved missense mutation in PKP2, the gene encoding plakophilin-2.

The case is a typical example of a mutation-dependent ARVC in a highly trained endurance athlete. The 12-lead ECG was manifestly abnormal with TWI in leads V1-V5 and localized right precordial QRS prolongation. The extent of the precordial TWI provides an indication of the extent of RV involvement and/or enlargement [19,22]. It has been suggested that patients with TWI in V1-V4 may have a more advanced stage of the disease [19]. TWI extending in the lateral leads (V5-V6) suggests LV involvement, which may occur early in the course of the disease [23]. In the athlete, however, CMR did not reveal any abnormalities suggesting involvement of the LV.

Another important finding on the ECG in this case was the presence of 2 premature ventricular contractions, which is considered abnormal in a $10 \mathrm{~s}$ ECG recording and should trigger further investigation [4]. In patients with RVOT tachycardia, a benign condition not associated with structural ventricular abnormalities, the QRS complex of the ventricular ectopy will invariably have an LBBB morphology that is positive in the inferior leads II, III, and AVF and almost always negative in aVL [19]. Therefore, the morphology of the ventricular ectopic beats with LBBB and superior axis (and positive in aVL) also hinted to a diagnosis of ARVC. Another ECG-criterion for distinction between idiopathic RVOT tachycardia and ARVC is provided by the QRS duration in lead I. Ainsworth et al. compared 24 RVOT tachycardia and 20 ARVC patients and reported that a QRS duration $>120$ milliseconds in lead I had a sensitivity of $100 \%$, specificity of $46 \%$, positive predictive value of $61 \%$, and negative predictive value of $100 \%$ for identifying those with ARVC [24]. Again, the presence of late potentials was indicative of ARVC since only a very small proportion (0$12 \%$ ) of RVOT patients has a positive SAECG versus $50 \%-$ $80 \%$ in those with ARVC [19].

\section{Case 4}

An asymptomatic 31 year old professional football player of Caucasian origin underwent ECG evaluation as part of preparticipation screening. The ECG (Fig. 5) revealed right ventricular hypertrophy voltage criteria $(\mathrm{R}-\mathrm{V} 1+\mathrm{S}-$ V5 $>10.5 \mathrm{~mm}$ and right axis deviation) and suspiciously low voltages in the limb leads. There were no T-wave changes. An echocardiogram showed a moderately dilated RV with normal systolic function and signs of mild pulmonary hypertension. Administration of agitated contrast suggested an intra-cardiac

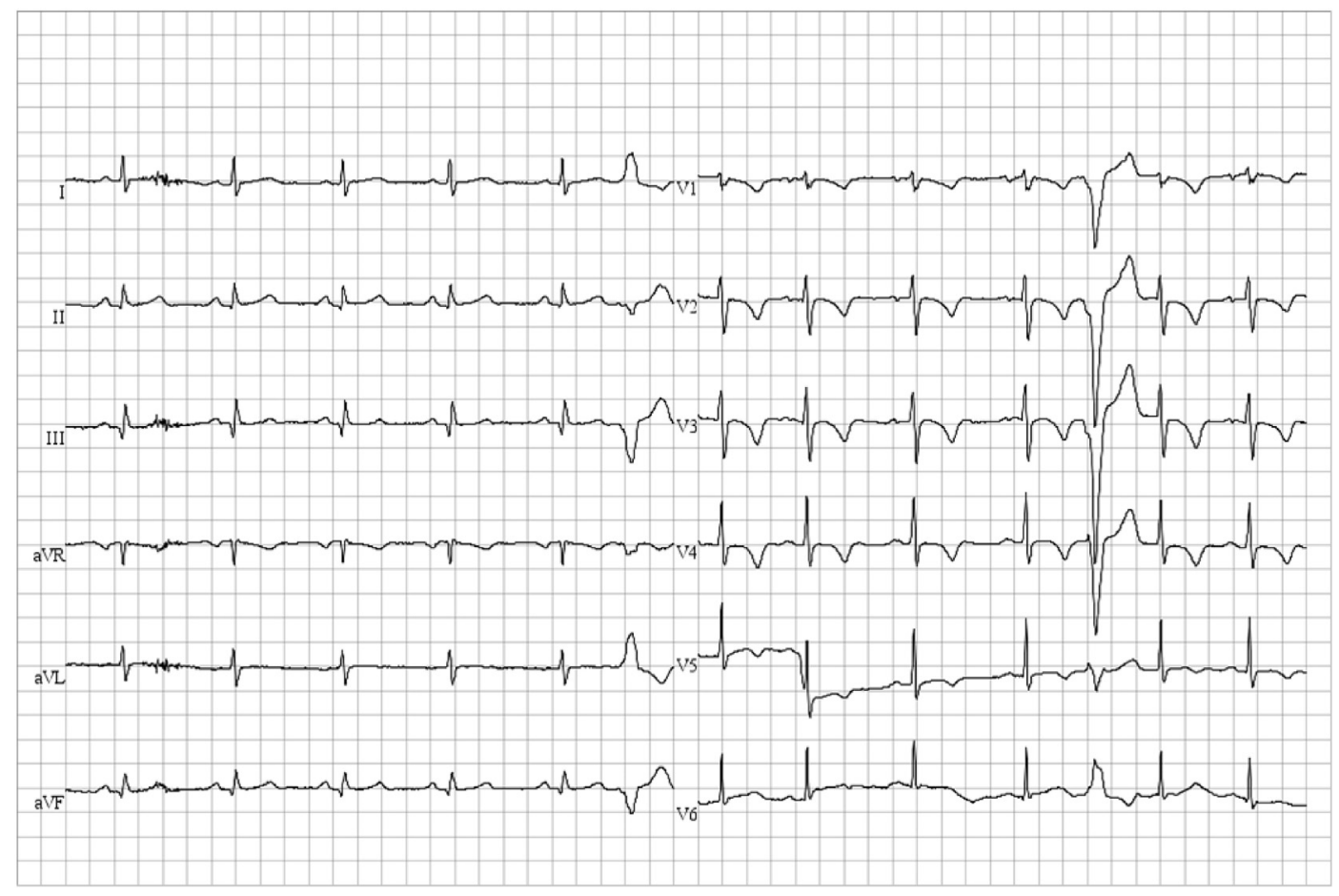

Fig. 4. Resting ECG in athlete 3. The ECG shows typical features of arrhythmogenic right ventricular cardiomyopathy with deep TWI in V1-V5, delayed S wave upstroke in V1 (>55 ms) and the presence of 2 ventricular ectopic beats with left bundle branch block morphology and superior axis. 


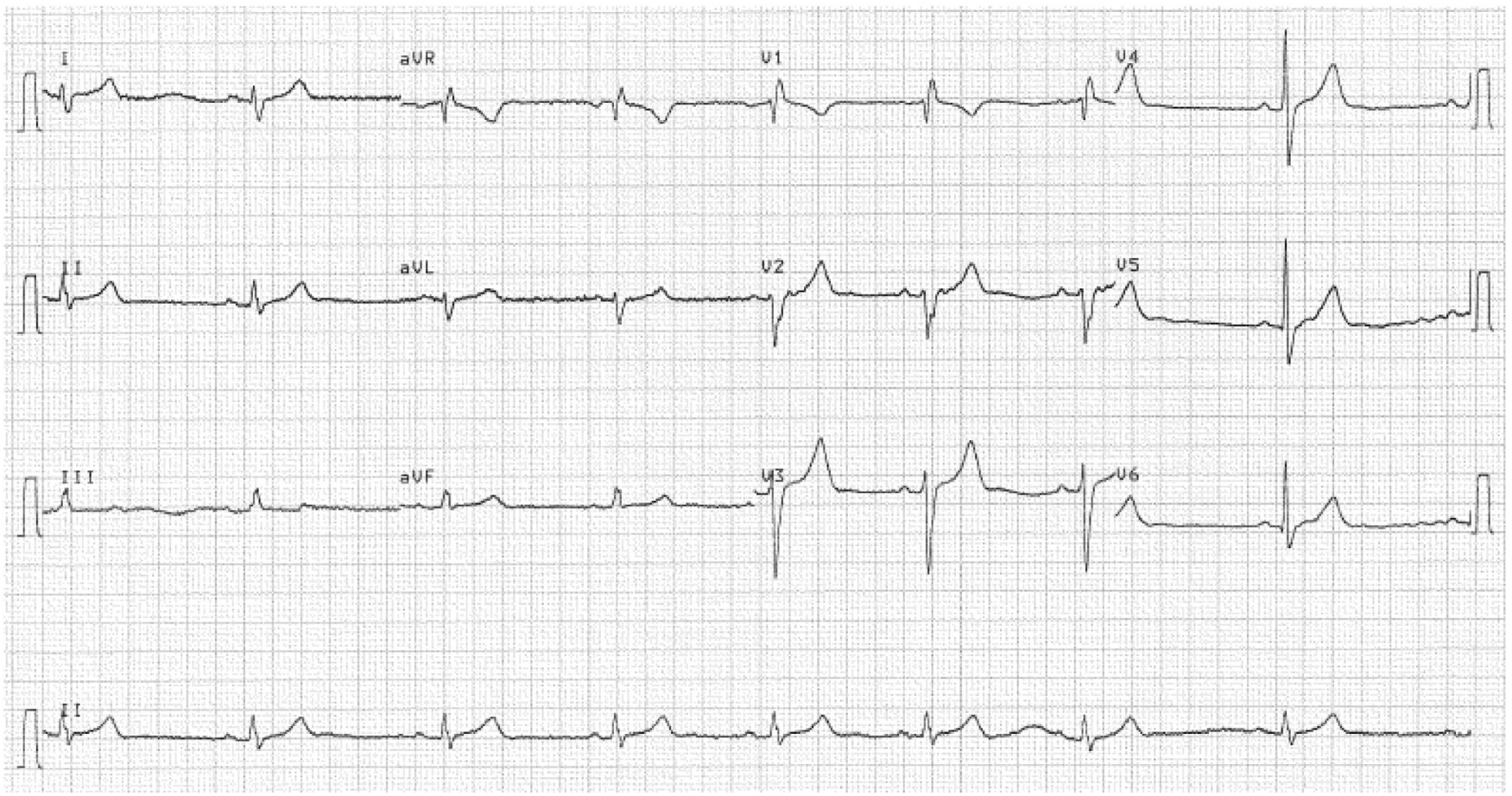

Fig. 5. Resting ECG in athlete 4. The ECG demonstrates right axis deviation, right ventricular hypertrophy on voltage criteria $(\mathrm{R}-\mathrm{V} 1+\mathrm{S}-\mathrm{V} 5>10.5 \mathrm{~mm}$ and right axis deviation) and low voltages in the limb leads.

shunt at the atrial level. Transesophageal echocardiography confirmed a moderate size sinus venous atrial septal defect $(1.1 \times 1.3 \mathrm{~cm})$ and the athlete was referred for open surgical repair. Follow-up imaging demonstrated complete regression of his RV dilation and mild pulmonary hypertension and he has now returned to professional football.

This case depicts another clinical scenario in which the load on the right heart is increased. Although the combination of right ventricular hypertrophy on voltage criteria and right axis deviation has been observed in up to $0.5 \%$ of young athletes in the absence of structural heart disease [25], both the ESC criteria and more recently proposed Seattle criteria for athlete ECG interpretation suggest that in combination, these findings are suspicious for the presence of pulmonary hypertension and should trigger further investigation in the athlete as the increased RV afterload may predispose athletes to the development of RV failure and increase the risk of sudden death $[3,13]$. Although the finding of low limb lead voltages is not discussed in either criteria, seen together with RVH and RAD the index of suspicion for the presence of underlying structural heart disease in this case was high. These ECG changes could be explained by the RV volume overload caused by the atrial septum defect. It is important to note that the hemodynamic impact varies considerable according to the underlying cause of congenital heart disease, even among patients with the same lesion. In fact, among patients with congenital heart disease the majority of individuals participating in exercise programs achieve significant improvement of their exercise capacity and psychological state [26]. Therefore, decisions regarding exercise prescription and sports participation should be tailored to each individual patient and only those who are likely to deteriorate as a consequence of regular physical exercise or those in whom exercise may trigger serious atrial/ventricular tachyarrhythmias should be restricted from sports participation [27].

\section{Conclusion}

The role of the ECG in the differentiation between physiological adaptations to endurance exercise and underlying RV pathology such as ARVC cannot be underestimated. Early recognition of ECG features of ARVC is important because sports continuation in an athlete with ARVC may trigger life threatening arrhythmias and cause progression of the arrhythmogenic substrate. In Caucasian endurance athletes, TWI in the right precordial leads is relatively common. However, TWI in leads V1-V3 should trigger comprehensive evaluation for ARVC (genetically dependent and/or due to the increased load of exercise). In black athletes, convex ST-segment elevation with biphasic TWI in leads V1-V4 is a common finding and considered benign. In contrast, symmetrical anterior TWI in V1-V3 preceded by isoelectric or downsloping ST segments is rare and should prompt further investigation to exclude ARVC. Early in the course of the disease, ECG findings related to ARVC can be subtle and overlap with those frequently observed in healthy endurance athletes. Therefore, suspicious symptoms in athletes with such minor diagnostic ECG-findings should prompt extensive evaluation including echocardiography, Holter monitoring, maximal exercise-ECG testing and possibly a cardiac MRI and electrophysiological study. Finally, the combination of voltage criteria for right ventricular hypertrophy and right axis deviation is another ECG hallmark of abnormal RV afterload 
and further evaluation for diagnosis and work-up of pulmonary hypertension should be considered.

\section{References}

[1] Pluim BM, Zwinderman AH, van der Laarse A, van der Wall EE. The athlete's heart. A meta-analysis of cardiac structure and function. Circulation 2000;101:336-44.

[2] Brosnan M, La Gerche A, Kalman J, Lo W, Fallon K, MacIsaac A, et al. Comparison of frequency of significant electrocardiographic abnormalities in endurance versus nonendurance athletes. Am J Cardiol 2014;113:1567-73.

[3] Corrado D, Pelliccia A, Heidbuchel H, Sharma S, Link M, Basso C, et al. Recommendations for interpretation of 12-lead electrocardiogram in the athlete. Eur Heart J 2010;31:243-59.

[4] Drezner JA, Ackerman MJ, Anderson J, Ashley E, Asplund CA, Baggish AL, et al. Electrocardiographic interpretation in athletes: the 'Seattle criteria'. Br J Sports Med 2013;47:122-4.

[5] La Gerche A, Heidbuchel H, Burns AT, Mooney DJ, Taylor AJ, Pfluger HB, et al. Disproportionate exercise load and remodeling of the athlete's right ventricle. Med Sci Sports Exerc 2011;43:974-81.

[6] Sawant AC, Bhonsale A, Te Riele AS, Tichnell C, Murray B, Russell SD, et al. Exercise has a disproportionate role in the pathogenesis of arrhythmogenic right ventricular dysplasia/cardiomyopathy in patients without desmosomal mutations. J Am Heart Assoc 2014;3.

[7] Heidbuchel H, Prior DL, La Gerche A. Ventricular arrhythmias associated with long-term endurance sports: what is the evidence? Br J Sports Med 2012;46(Suppl 1):i44-50.

[8] Basso C, Corrado D, Marcus FI, Nava A, Thiene G. Arrhythmogenic right ventricular cardiomyopathy. Lancet 2009;373:1289-300.

[9] La Gerche A, Robberecht C, Kuiperi C, Nuyens D, Willems R, de Ravel $\mathrm{T}$, et al. Lower than expected desmosomal gene mutation prevalence in endurance athletes with complex ventricular arrhythmias of right ventricular origin. Heart 2010;96:1268-74.

[10] Nasir K, Bomma C, Tandri H, Roguin A, Dalal D, Prakasa K, et al. Electrocardiographic features of arrhythmogenic right ventricular dysplasia/cardiomyopathy according to disease severity: a need to broaden diagnostic criteria. Circulation 2004;110:1527-34.

[11] Steriotis AK, Bauce B, Daliento L, Rigato I, Mazzotti E, Folino AF, et al. Electrocardiographic pattern in arrhythmogenic right ventricular cardiomyopathy. Am J Cardiol 2009;103:1302-8.

[12] Marcus FI, McKenna WJ, Sherrill D, Basso C, Bauce B, Bluemke DA, et al. Diagnosis of arrhythmogenic right ventricular cardiomyopathy/ dysplasia: proposed modification of the task force criteria. Eur Heart J 2010;31:806-14.

[13] Drezner JA, Ashley E, Baggish AL, Borjesson M, Corrado D, Owens DS, et al. Abnormal electrocardiographic findings in athletes: recognising changes suggestive of cardiomyopathy. Br J Sports Med 2013;47:137-52.

[14] Simonneau G, Gatzoulis MA, Adatia I, Celermajer D, Denton C, Ghofrani A, et al. Updated clinical classification of pulmonary hypertension. J Am Coll Cardiol 2013;62:D34-41.
[15] Rich S, Dantzker DR, Ayres SM, Bergofsky EH, Brundage BH, Detre KM, et al. Primary pulmonary hypertension. A national prospective study. Ann Intern Med 1987;107:216-23

[16] Zaidi A, Ghani S, Sheikh N, Gati S, Bastiaenen R, Madden B, et al. Clinical significance of electrocardiographic right ventricular hypertrophy in athletes: comparison with arrhythmogenic right ventricular cardiomyopathy and pulmonary hypertension. Eur Heart J 2013;34:3649-56.

[17] Jain R, Dalal D, Daly A, Tichnell C, James C, Evenson A, et al. Electrocardiographic features of arrhythmogenic right ventricular dysplasia. Circulation 2009;120:477-87.

[18] Zaidi A, Ghani S, Sharma R, Oxborough D, Panoulas VF, Sheikh N, et al. Physiological right ventricular adaptation in elite athletes of African and Afro-Caribbean origin. Circulation 2013;127:1783-92.

[19] Marcus FI, Zareba W. The electrocardiogram in right ventricular cardiomyopathy/dysplasia. How can the electrocardiogram assist in understanding the pathologic and functional changes of the heart in this disease? J Electrocardiol 2009;42 [136 e131-135].

[20] Heidbuchel H, Hoogsteen J, Fagard R, Vanhees L, Ector H, Willems R, et al. High prevalence of right ventricular involvement in endurance athletes with ventricular arrhythmias. Role of an electrophysiologic study in risk stratification. Eur Heart J 2003;24:1473-80.

[21] James CA, Bhonsale A, Tichnell C, Murray B, Russell SD, Tandri H, et al. Exercise increases age-related penetrance and arrhythmic risk in arrhythmogenic right ventricular dysplasia/cardiomyopathy-associated desmosomal mutation carriers. J Am Coll Cardiol 2013;62:1290-7.

[22] Nava A, Canciani B, Buja G, Martini B, Daliento L, Scognamiglio R, et al. Electrovectorcardiographic study of negative $t$ waves on precordia leads in arrhythmogenic right ventricular dysplasia: relationship with right ventricular volumes. J Electrocardiol 1988;21:239-45.

[23] Sen-Chowdhry S, Syrris P, Prasad SK, Hughes SE, Merrifield R, Ward D, et al. Left-dominant arrhythmogenic cardiomyopathy: an under-recognized clinical entity. J Am Coll Cardiol 2008;52:2175-87.

[24] Ainsworth CD, Skanes AC, Klein GJ, Gula LJ, Yee R, Krahn AD. Differentiating arrhythmogenic right ventricular cardiomyopathy from right ventricular outflow tract ventricular tachycardia using multilead QRS duration and axis. Heart Rhythm 2006;3:416-23.

[25] Brosnan M, La Gerche A, Kalman J, Lo W, Fallon K, MacIsaac A, et al. The Seattle criteria increase the specificity of preparticipation ECG screening among elite athletes. Br J Sports Med 2014;48:1144-50.

[26] Dua JS, Cooper AR, Fox KR, Graham Stuart A. Exercise training in adults with congenital heart disease: feasibility and benefits. Int J Cardiol 2010;138:196-205.

[27] Pelliccia A, Fagard R, Bjornstad HH, Anastassakis A, Arbustini E, Assanelli D, et al. Recommendations for competitive sports participation in athletes with cardiovascular disease: a consensus document from the study group of sports cardiology of the working group of cardiac rehabilitation and exercise physiology and the working group of myocardial and pericardial diseases of the European Society of cardiology. Eur Heart J 2005;26:1422-45. 